\title{
Swantje Piotrowski
}

\section{Kieler Professoren, ihre Söhne und der linke Terrorismus}

\section{Zum Umgang mit akademischem Traditionsbewusstsein und politischer Radikalisierung an der CAU}

\begin{abstract}
„Nicht achten kann ich diejenigen, die bei anderen ihre Unzufriedenheit mit meiner Amtsführung äußern, es aber nicht wagen, mir das auch zu sagen. Verachten kann ich nur diejenigen - es sind nicht nur Kollegen des Lehrkörpers -, die den hochschulpolitischen Dissens in die private Sphäre übertragen und genußvoll eine Reihe kleiner niederträchtiger Lügen über meine Familie erfinden bzw. weiterverbreiten. “
\end{abstract}

Mit diesen Worten über seine Zeit als Rektor legte Professor Ludwig Weisbecker ${ }^{2}$ am 1. April sein Amt für das akademische Jahr 1969/70 nieder und rechnete einmal mehr mit seinen Kritikern, seinen Kolleginnen und Kollegen sowie dem gesamten Konsistorium ab: „Es gibt eine nicht geringe Anzahl von Kollegen [...], mit denen bei aller Toleranz eine Zusammenarbeit unmöglich ist“, $\underline{\underline{3}}$ fuhr Weisbecker fort und übergab scheinbar voller Verbitterung nach „langen und harten Auseinandersetzungen“ das Rektorat an seinen Nachfolger Diedrich Wilhelm Schröder, Professor für Pflanzenernährung und Bodenkunde. ${ }^{4}$ Die harten Auseinandersetzungen, auf die Weisbecker sich hier bezieht, waren die Folge der Unruhen und Spannungen, die sich im Zuge der studentischen Protestbewegung nicht nur bundesweit - ausgehend von den tödlichen Schüssen auf den Studenten Benno Ohnesorg am 2. Juni 1967 und das Attentat auf Rudi Dutschke am 11. April 1968 -, sondern auch an der Kieler Hochschule immer stärker ausgebreitet hatten. Die sogenannte 68er-Bewegung, die sich aus den Kindern der Nachkriegs- und Aufbaugeneration zusammensetzte, hatte sich mit zahlreichen öffentlichkeitswirksamen Demonstrationen von den Lebensentwürfen und Karrierebahnen ihrer Eltern distanziert. $\underline{5}$ Neben dem allgemeinen Misstrauen gegenüber dem Staat und der Gesellschaft mit ihren als antiquiert betrachteten Normen und Werten kam es in Kiel vor allem durch zwei weitere Themenkomplexe zu Dynamisierungsschüben innerhalb der Protestbewegung: durch die geplante Immatrikulationsverordnung des schleswig-holsteinischen Kultusministeriums aus dem Jahr 1968, die als Maßnahme gegen stetig wachsende Studierendenzahlen gedacht war und zu einer Zwangsexmatrikulation von Langzeitstudierenden geführt hätte, und durch die Diskussionen um ein neues Hochschulgesetz. ${ }^{6}$ 


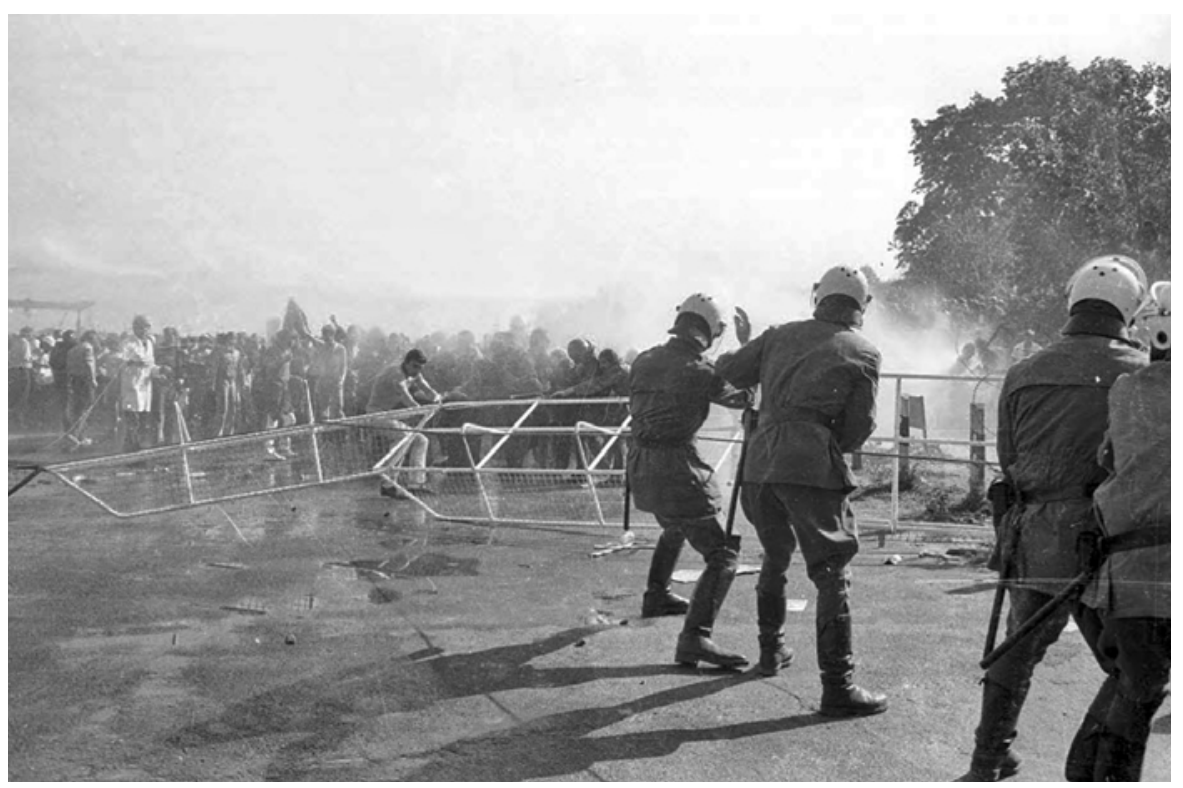

Abb. 1: Demonstration gegen neues Hochschulgesetz und Ordnungsrecht (9.6.1969).

(C) 1969 Friedrich Magnussen, veröffentlicht unter der Lizenz CC-BY-SA 3.0 DE,

Stadtarchiv Kiel, Sign. 21.930.

Bereits im Jahr 1962 hatten die bundesdeutschen Debatten und Kontroversen um die neue Rechtsordnung der Hochschule begonnen, die schlussendlich im Mai 1973 in Kraft trat und deren sichtbarste Konsequenz die Ablösung der Rektoratsverwaltung durch eine Präsidialverwaltung war. Beide geplanten Maßnahmen führten zu heftigen Auseinandersetzungen und tiefen Verwerfungen zwischen den Studierenden und der Hochschulverwaltung der Christian-Albrechts-Universität zu Kiel (CAU).

So informierten die ,Mitteilungen des Rektors' über elf besondere Vorkommnisse allein in den ersten Monaten des Wintersemesters 1969/70, die sich überwiegend auf Störungen oder gar Verhinderungen von Lehrveranstaltungen bezogen. $\stackrel{乛}{ }$ Unter den weiteren zahlreichen Aktionen der linken Protestbewegung können sicher die Besetzung des Seminars für Wissenschaft und Geschichte der Politik im achten Stock des Universitätshochhauses und die Auseinandersetzungen mit dessen Direktor Michael Freund als einer der Höhepunkte des Jahres 1969 angesehen werden. Die Vertreter der studentischen Linken hatten auf die Vorwürfe des Rektorats hin betont, dass in der Auseinandersetzung mit der herrschenden Gewalt Gegengewalt notwendig sei.․․ In Folge der zunehmenden Eskalation hatten am 11. November 196932 Professo- 
ren der Philosophischen Fakultät eine Dienstaufsichtsbeschwerde gegen Rektor Weisbecker beim Kultusministerium eingereicht, mit dem Vorwurf, dieser habe sich nicht energisch genug für einen störungsfreien Ablauf von Lehre, Forschung und Tätigkeit der Hochschulselbstverwaltungsorgane eingesetzt. 10 Ihren vorzeitigen Höhepunkt erreichten die Konflikte um das Rektorat und die Person Weisbeckers in der am 25. November 1969 abgehaltenen Senatssitzung, die durch Studierende so massiv gestört wurde, dass Weisbecker die Sitzung beenden musste. Bei einer Rangelei an der Tür erhielt Weisbecker daraufhin einen Schlag in den Unterleib und brach mit einem Kreislaufschock bewusstlos zusammen.르 Der sogenannte „Knockout unter die Gürtellinie“ legte nicht nur Weisbecker, sondern den gesamten Universitäts- und Lehrbetrieb für eine halbe Woche lahm. $\frac{12}{}$

Doch Weisbecker verzichtete auf einen Strafantrag: Er sei, so sagte er, ,entschlossen so zu tun, als ob nichts gewesen wäre“. $\frac{13}{}$ Damit folgte er den Maximen seines Rektoratsjahrs, das auf Deeskalation ausgerichtet war, auf ein „Überleiten von Emotionen auf einfache, durchschaubare evolutive Konzeptionen“. ${ }^{14}$ In einem Kieler Nachrichten-Interview vom 22. März 1969 hatte sich Weisbecker bei seinem Amtsantritt auf die Seite der Studentenbewegung gestellt: $\frac{15}{5}$ Er sei gegen verkrustete Universitätsstrukturen, gegen eine Wissenschaft, die ihre Forschungsergebnisse nicht einer breiten Öffentlichkeit zugänglich mache. Allerdings wandte er sich gegen die oft gewaltsamen Methoden, mit denen die Protestler Veränderungen durchzusetzen versuchten. Er führte diese Linie der Deeskalation konsequent bis zum Ende seines Amtsjahres weiter. Weisbecker wollte mit den Ereignissen rund um die studentische Protestbewegung keine Schlagzeilen machen. Er sagte: „Das hätte die wenigen positiven Ansätze einer sinnvollen Evolution im Keim erstickt, die Universität den Rest an Autonomie gekostet und den Staat zum Eingreifen gezwungen - so sehr das auch von manchen Mitgliedern mancher Gruppen gewünscht“ wurde. $\frac{16}{}$

Ludwig Weisbecker saß mit seiner Haltung in gewisser Weise zwischen den Stühlen. Durch das Amt des Rektors an akademische und tradierte Normen gebunden, musste er sich den spannungs- und konfliktreichen Verhältnissen im Sinne der noch in weiten Zügen bestehenden Ordinarienuniversität gegenüber verantwortlich zeichnen,,$^{17}$ bekundete jedoch immer wieder Sympathien gegenüber der antiautoritären Bewegung.

Die hier kurz skizzierten Ereignisse des Rektoratsjahres 1969/70 zeigen das Spannungsfeld zwischen Studentenrevolte und der um ihre akademische Selbstbestimmung fürchtende Professorenschaft auf. Die Christiana Albertina, bis dahin als traditionsbewusste Ordinarienuniversität von einem mehrheitlich männlichen Kollegium dominiert, befand sich durch die Pläne einer neuen Hochschul- und Studienreform im Prozess eines vermeintlichen Autonomieverlustes. Die Professoren fürchteten zuneh- 


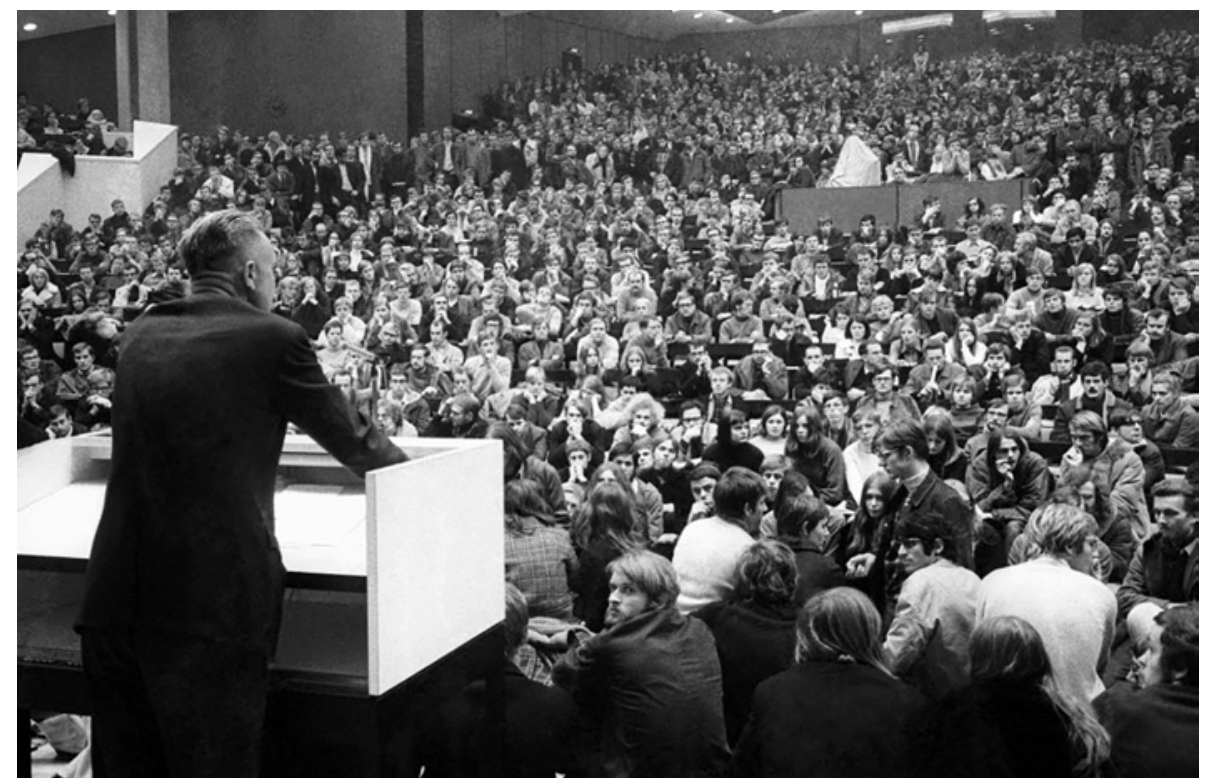

Abb. 2: Studentisches Teach-in im überfüllten Audimax der CAU (27.11.1969). (C) 1969 Friedrich Magnussen, veröffentlicht unter der Lizenz CC-BY-SA 3.0 DE, Stadtarchiv Kiel, Sign. 21.909.

mend um ihre akademische Selbstbestimmung; zum einen in der Abgrenzung zum Kultusministerium, zum anderen gegenüber den Studierenden..$\underline{18}$

Neben diesen konkret im universitären Kontext erfahrbaren institutionellen Problemen auf der einen Seite, die für die vorliegende Untersuchung auf einer regionalen Ebene verortet wurden, trat eine weitere Perspektive hinzu: Durch die tödlichen Schüsse auf die Söhne der Kieler Professoren Georg von Rauch, Professor für Osteuropäische Geschichte, und Ludwig Weisbecker in den Jahren 1971/72 trat der überregionale linke Terrorismus in den regionalen Raum der Christiana Albertina ein.

Im Verlauf der nun folgenden Untersuchung soll gezielt den Fragen nachgegangen werden, wie sich die CAU - in der vorliegenden Untersuchung durch die verfassungsmäßigen Organe des Rektorats und der Universitäts-Pressestelle repräsentiert - zum einen auf der regionalen Ebene positionierte, das heißt wie sie als Trägerin akademischer Werte und Normen mit der politischen Radikalisierung in ihrem direkten Umfeld umging, und zum anderen, wie sie den tragischen Ereignissen rund um den Tod der Kieler Professorensöhne und damit der bundesweiten politischen Radikalisierung be- 


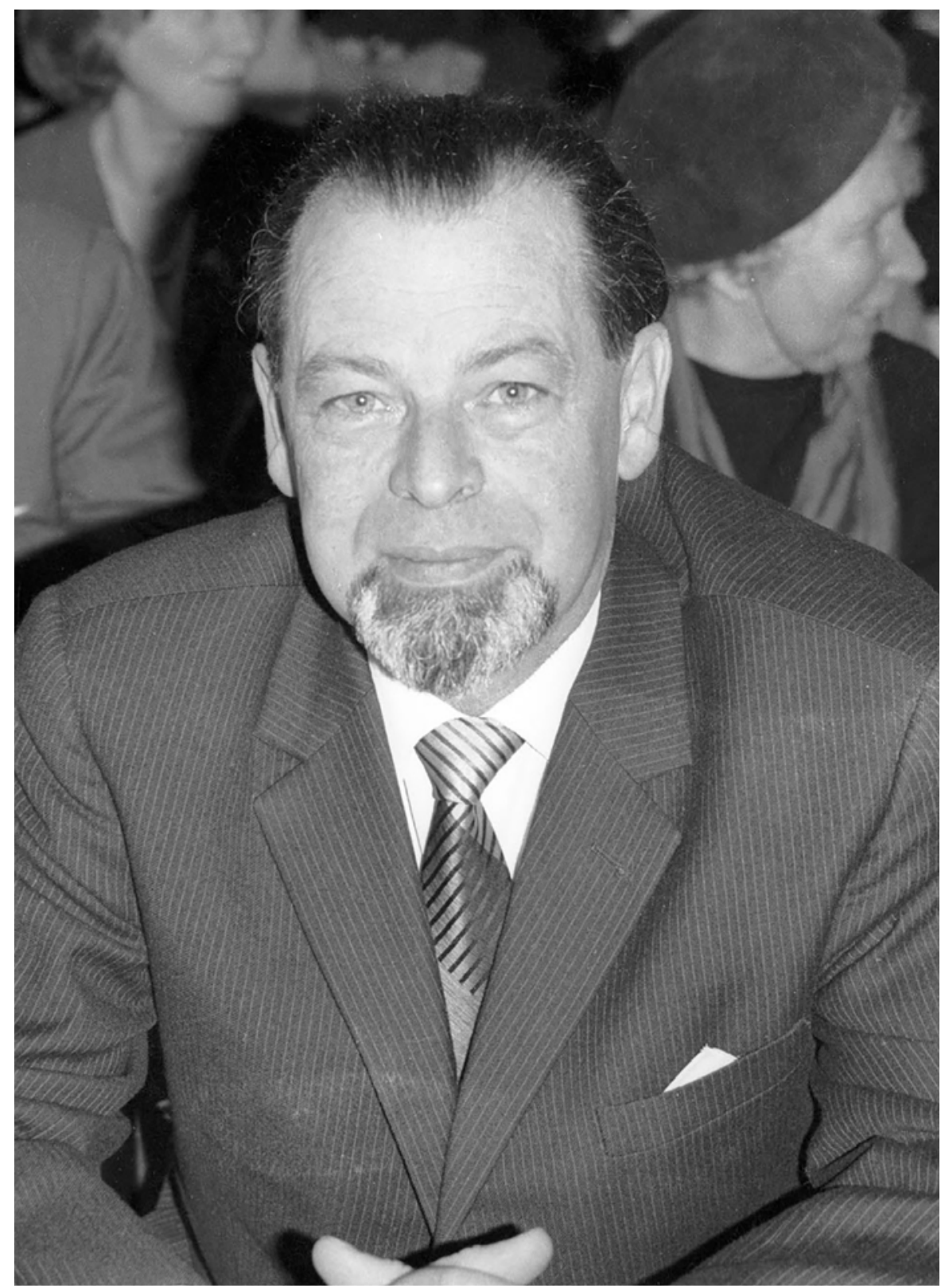

Abb. 3: Prof. Dr. Ludwig Weisbecker.

(C) 1968 Friedrich Magnussen, veröffentlicht unter der Lizenz CC-BY-SA 3.0 DE, Stadtarchiv Kiel, Sign. 42.525 
gegnete. Welche Haltung nahm das Rektorat der CAU gegenüber der Öffentlichkeit, den Angehörigen und den Medien ein? In welcher Form äußerte es sich zu den Geschehnissen und in welchem Maß trug es zu einer Aufklärung und Transparenz der Umstände bei?

Im Folgenden werden zur Beantwortung der oben aufgeworfenen Fragen zunächst die Biographien der beiden Kieler Professoren vorgestellt und im direkten Anschluss kurz das jeweilige Verhältnis der Väter zu ihren Söhnen skizziert. Um die Relevanz der tragischen Ereignisse um den gewaltsamen Tod der Söhne im Kontext der Fragestellungen zu verdeutlichen und für den weiteren Verlauf der Argumentation einbinden zu können, werden zudem die Biographien der Söhne und ihr Weg in den Untergrund dokumentiert. Es folgt ein Blick auf die überregionalen Reaktionen bezüglich der tödlichen Schüsse, um dann anhand ausgewählter Quellen die Rolle der CAU in den Krisenjahren 1969 bis 1972 zu verorten.

\section{Ludwig Weisbecker und Georg von Rauch und das Verhältnis zu ihren Söhnen}

Ludwig Weisbecker war von 1962 bis zu seinem Tod 1979 Professor für Innere Medizin und Direktor der 2. Medizinischen Klinik und Poliklinik in Kiel. Am 1. April trat er, wie bereits geschildert, sein Amt als Rektor der CAU für das Rektoratsjahr $1969 / 70$ an.

Geboren wurde Weisbecker am 19. März 1915 in Mainz, wo er 1933 sein Abitur ablegte und das Studium der Medizin und Chemie begann. Nach wechselnden Studienorten promovierte er 1938 an der Universität Freiburg zum Doktor der Medizin. Da ihm aufgrund seiner jüdischen Herkunft - sein Vater war Jude - die ärztliche Approbation verweigert wurde, arbeitete er zunächst als Pharmakologe bei den Chemischen Werken Albert in Wiesbaden. ${ }^{19}$ Das Arbeitszeugnis, das Weisbecker bei seiner Entlassung erhalten hatte, gibt Auskunft darüber, dass er aus politischen Gründen und auf höhere Anweisung das Werk im April 1943 verlassen musste..$^{20}$ Nach kurzer Haft im Mainzer Gestapo-Gefängnis kam er am 5. April 1943 in das Arbeitserziehungslager in Frankfurt Heddernheim. Am 19. Mai wurde er mit dem Vermerk „Politischer Mischling 1. Grades“ in das Konzentrationslager Buchenwald eingeliefert. 리 Dort, in Haft gehalten, arbeitete Weisbecker bis Kriegsende als Lagerarzt und übernahm am 11. April

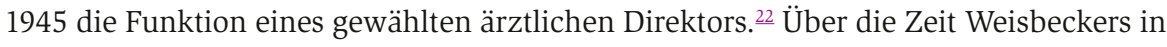
Buchenwald, so schreibt Karin König, gibt es nur wenige Dokumente. Im Prozess gegen die Führung des Konzentrationslagers trat Weisbecker 1947 als Zeuge auf. „Dort sagte er zur Tätigkeit des SS Arztes Dr. Bender aus und entlastete ihn [...]. “르 Auch der Vater Weisbeckers, Louis Weisbecker, wurde im April 1943 ohne Angaben von Grün- 
den oder eines Verfahrens von der Gestapo verhaftet und zunächst im Konzentrationslager Auschwitz, später in Mauthausen inhaftiert. $\underline{\underline{24}}$

Weisbecker setzte bereits 1946 seine akademische Karriere in Freiburg i. Br. fort, zunächst als wissenschaftlicher Assistent, ab 1948 als Privatdozent und ab 1953 als außerordentlicher Professor der Pharmakologie. Im April 1946 heiratete Weisbecker Dr. Rotraud Röttger, mit der er drei gemeinsame Kinder hatte. ${ }^{25}$ Im Jahr 1962 folgte Weisbecker dann dem Ruf als ordentlicher Professor für Innere Medizin an die CAU. $\underline{26}$ Nach dem Tod seines Sohnes im Jahr 1972 erkrankte Ludwig Weisbecker schwer. „Mit Rücksicht auf meine schwer angeschlagene Gesundheit“, so schreibt Weisbecker an den Kultusminister Walter Braun bereits im März 1979, „bitte ich darum, mich mit vollendetem 65. Lebensjahr zu emeritieren. “ Weisbecker sehe „keine Chance, daß [er] noch lange dienstfähig bleibe.“르 Am 13. Juni 1979, noch vor seiner Emeritierung, starb Ludwig Weisbecker in Mielkendorf.

Das Verhältnis zwischen Ludwig Weisbecker und seinem Sohn Thomas war nicht frei von Konflikten..$^{28}$ In ihren Ausführungen, die sich auf die Aussagen von Thomas' Schwester, Verena Weisbecker, berufen, waren es die vielen Kontraste in den Biographien von Vater und Sohn, die ihre Beziehung belasteten: Ludwig Weisbecker, der jüdische KZ-Überlebende, genoss seine spätere berufliche und gesellschaftliche Anerkennung; er war angepasst und politisch konservativ. ${ }^{29}$ Im Gegensatz zur Mutter zeigte er keinerlei Verständnis für das Verhalten seines Sohnes.

„Es störte ihn [Ludwig Weisbecker], daß die Kinder geworden waren, wie er gewesen war [...]. Die Kinder hatten von klein an in ihm den Helden gesehen, der aktiv gegen den Nationalsozialismus gekämpft hat, der jahrelang im KZ gewesen war. Es irritierte sie, daß er nun nichts mehr sein wollte als hervorragend in seinem Beruf und geachtet in der Gesellschaft. “으

Nach dem Umzug von Thomas Weisbecker nach West-Berlin im Jahr 1969 kam es zu einer weiteren Verschlechterung des Vater-Sohn-Verhältnisses. Fortan musste er dem Elternhaus fernbleiben, nach seinem Tod wurde sein Name innerhalb der Familie verschwiegen. $\frac{31}{11}$

Trotz der aufgezeigten Spannungen zwischen Vater und Sohn, dürften die Biographie und der berufliche Werdegang Ludwig Weisbeckers keinen ausschlaggebenden Grund für die Radikalisierung des Sohnes geboten haben. Die Familiengeschichte der Weisbeckers bot zur Überwindung des Traumas Faschismus keine Rückbezüge zu eventuellen Tätern, im Gegenteil, von einem überkommenen neuen Faschismus, den die militante linksradikale Szene als stete Gefahr ansah, konnte hier nicht die Rede sein. 


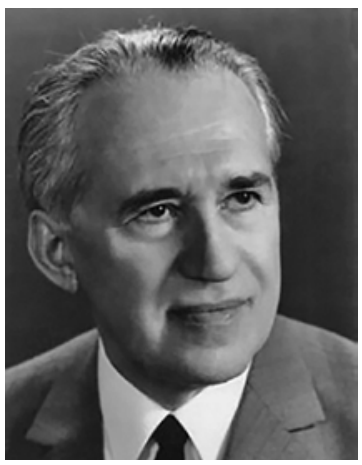

Abb. 4: Prof. Dr. Georg von

Rauch. Alle Rechte vorbehalten (Ostdeutsche Biographie, Kulturportal West-Ost), Quelle: https://kulturportalwest-ost.eu/.
Georg von Rauch wurde am 13. August 1904 in Pskow (Pleskau), Russland, als Sohn von Dr. med. Cornelius und Frieda von Rauch (geb. Brock) geboren. $\frac{32}{2}$ Nach seinem Abitur 1922 studierte er Geschichte und Philosophie an den Universitäten Dorpat, Tübingen und Breslau und ging dann zunächst von 1927 bis 1936 in den Schuldienst. Im Jahr 1938 heiratete von Rauch Margarethe Reimer, gemeinsam hatten sie drei Kinder. Ab 1941 - zu diesem Zeitpunkt war von Rauch bereits Privatdozent arbeitete er für die Fremden Heere Ost (FHO). $\underline{33}$ Als Russland-Kenner und Ostwissenschaftler waren seine Tätigkeiten innerhalb der deutschen Wehrmacht hochgeschätzt: Er übersetzte abgefangene Feldpostbriefe, Befehle der Führung der Roten Armee, Flugblätter sowie Reden Stalins. Nach seiner amerikanischen Kriegsgefangenschaft lehrte er ab 1946 als Privatdozent, ab 1956 als außerordentlicher Professor für Osteuropäische Geschichte an der Phillips-Universität Marburg. Im Jahr 1958 folgte er dem Ruf an die CAU als ordentlicher Professor für Osteuropäische Geschichte und gründete gleichzeitig das Seminar für Osteuropäische Geschichte in Kiel.

Bis 1972 und damit bis zum Jahr seiner Emeritierung blieb von Rauch dem Historischen Seminar treu; er starb am 17. Oktober 1991 in Kiel. In zahlreichen Nachrufen, unter anderem im Jahrbuch des Bundesinstituts für ostdeutsche Kultur und Geschichte, heißt es:

„Herkunft und Lebensweg ließen Georg von Rauch zum Vermittler zwischen den Völkern Ostmitteleuropas werden. In Wort und Schrift gelang es dem Forscher und Lehrer wie nur wenigen anderen, viele Jahrzehnte lang auch über den akademischen Bereich hinaus anzuregen und schöpferisch zu wirken. Toleranz und menschliche Wärme, verbunden mit kritischer Aufgeschlossenheit, bestimmten die Begegnungen mit diesem Gelehrten, dessen Vorbild lange nachwirken wird. “쎠

Anlässlich des 100. Todestages Georg von Rauchs fand am 9. August 2004 eine internationale Historikerkonferenz in Pskow statt.

Obwohl Georg von Rauch jun. gute Gründe gehabt hätte, die politische Vergangenheit seines Vaters in Frage zu stellen, wurde das Verhältnis der beiden als gut beschrieben. Die Publikationen seines Vaters zur Sowjetunion schätzte der Sohn zwar als konservativ, aber lesenswert ein. Im Gegensatz zur Familie Weisbecker scheint sich 
die Familie von Rauch auch in der Zeit, in der sich Georg zusehends radikalisierte, vor Gericht stand, in den Untergrund ging und schließlich erschossen wurde, nie von ihm zu distanzieren. $\underline{35}$

\section{Die Biographien der Söhne und ihr Weg in den Untergrund}

Die Professorensöhne Thomas Weisbecker und Georg von Rauch jun. gehörten bereits zu Lebzeiten zu den Stars der linksradikalen Szene in West-Berlin. Nach ihrem gewaltsamen Tod wurden sie dann schnell zu bundesweit bewunderten Ikonen des bewaffneten Kampfes stilisiert. ${ }^{36}$ Ihre Biographien sind eng mit bestimmten Dynamisierungsschüben der linksradikalen Subkulturen verbunden: So waren beide Aktivisten der ,Kommune 1‘, der ,Umherschweifenden Haschrebellen', der ,Tupamaros West-Berlin', dem sogenannten ,Berliner Blues‘ sowie schlussendlich auch der ,Roten Armee Fraktion (RAF) ‘. 37 Damit waren sie aktiv an der Entstehung und den Transformationsprozessen des linken Terrorismus beteiligt, $\underline{\underline{38}}$ und ihr Tod lieferte der weiteren Entwicklung von Radikalisierung und Entgrenzung der Gewalt eine neue Legitimationsgrundlage. $\frac{39}{9}$

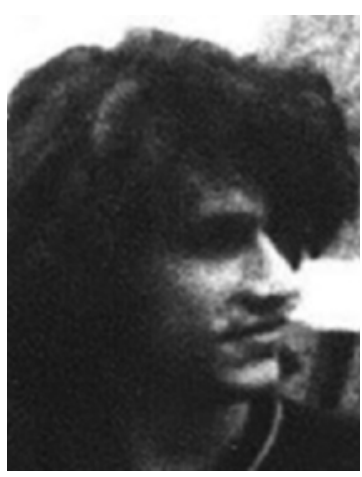

Abb. 5: Georg von Rauch jun. (C) Tommy-Weisbecker-Haus, veröffentlicht unter der Lizenz CC-BY-NC 3.0 DE.

Georg von Rauch, am 12. Mai 1947 in Marburg geboren, und Thomas Weisbecker, am 24. Februar 1949 in Freiburg i. Br. geboren, verbrachten beide ihr Jugend in Kiel. Sie gingen auf dasselbe Gymnasium, die Kieler Gelehrtenschule, und waren beide Professorensöhne. Der weitere Verlauf ihrer Biographien zeigte im Vergleich zu anderen Radikalisierungsprozessen keine signifikanten Unterschiede. Eine sozialwissenschaftliche Untersuchung des Bundesinnenministeriums zum bundesdeutschen Terrorismus, die direkt nach der Schleyer-Entführung im Jahr 1978 in Auftrag gegeben worden war, zeichnete in einer Datenerhebung von 250 Personen, die überwiegend aus den linksextremen Gruppierungen stammten, demnach folgendes Bild:

„Die Väter von 47 Prozent der Terroristen kamen im Gegensatz zu 12 Prozent im Bevölkerungsdurchschnitt aus gehobenen Berufen. Es waren vornehmlich die Kinder von Architekten, Ärzten, Pastoren, Richtern, Sparkassendirektoren, Universitätsprofessoren und anderen angesehenen Berufsgruppen. Hinzu kam im linken Terrorismus ein überdurchschnittliches Bildungsniveau. Im Gegensatz zu 19 Prozent im Bevölkerungsdurchschnitt hatten 47 Prozent aller Terroristen Abitur und eine Hochschule oder Universität besucht. “0ㅜ 


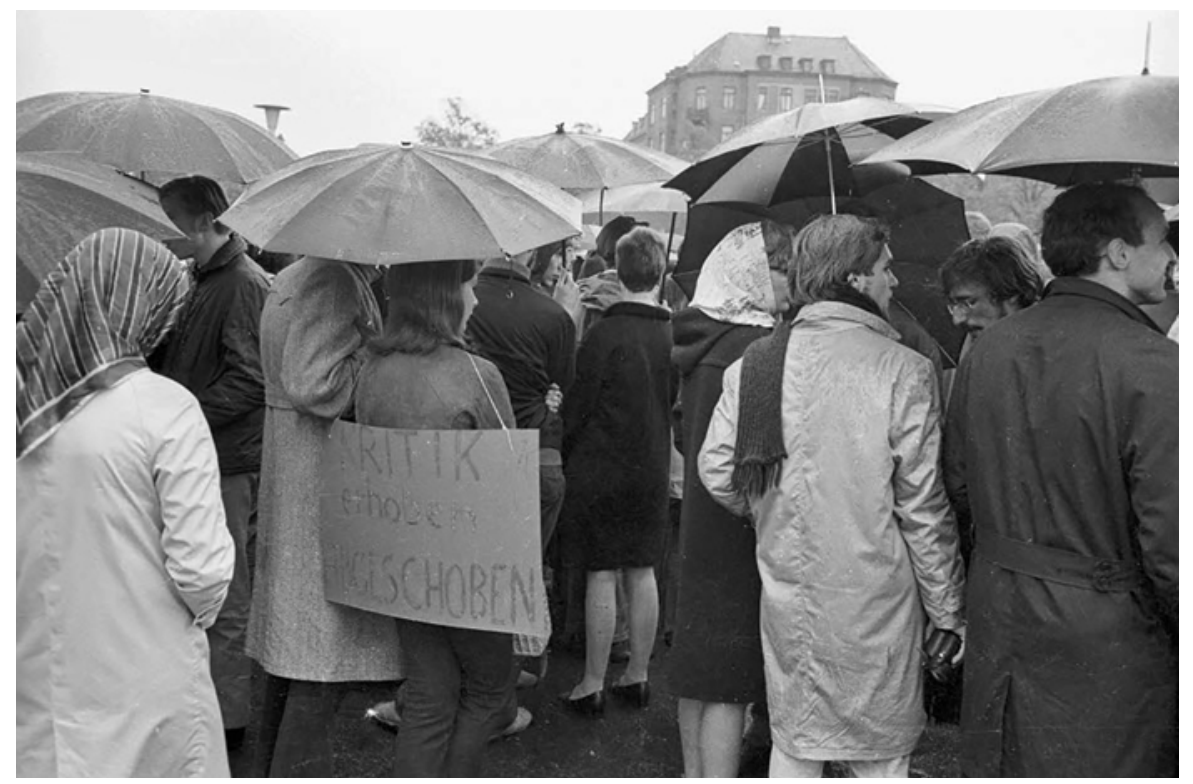

Abb. 6: Demonstration gegen den Verweis zweier Schüler von der Gelehrtenschule auf dem Exerzierplatz (9.10.1968). ( 1968 Friedrich Magnussen, veröffentlicht unter der Lizenz CC-BY-SA 3.0 DE, Stadtarchiv Kiel, Sign. 21.594.

In der Ansprache zu Georg von Rauchs jun. Beerdigung heißt es:

„Es ist ja einfach albern, leichtfertig und höhnisch, wenn immer wieder gesagt und geschrieben wird, Georg von Rauch - ausgerechnet ein Professorensohn; Gudrun Ensslin - ausgerechnet eine Pastorentochter [...]. Ja, gerade weil sie mit einer dynamischen Intelligenz begabt sind, weil sie erzogen worden sind zu einem unbestechlichen Rechtsdenken, sind sie auf den Weg der kompromißlosen Konsequenz gekommen, an dessen Ende wir für Georg von Rauch

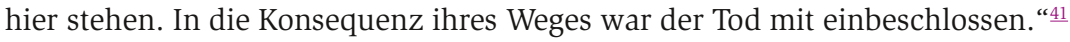

Ob es nun die zitierte „kompromißlose Konsequenz“ der beiden Söhne war, die sie in die Radikalisierung trieb, bleibt an dieser Stelle offen. Zumindest war es zwangsläufig ein Ausbruch der Söhne aus den ihnen vorgegebenen Lebensentwürfen und Karrierebahnen ihrer Väter. Die beiden Kieler Professoren waren über ihr akademisches Wirken an der CAU hinaus wichtige Repräsentanten bundesdeutscher Nachkriegseliten. ${ }^{42}$ Ihnen galt nicht nur die fundamentale Misstrauenserklärung ihrer Söhne, sondern noch dazu einer ganzen jüngeren Generation. Die tradierten Normen und Werte 
der Nachkriegsgesellschaft wurden in Frage gestellt, gerade im Hinblick auf eine in ihren Augen unzureichende Aufklärung der NS-Vergangenheit und die als schockierend empfundenen Verhältnisse rund um den Vietnamkrieg. Diese Umstände ließen die Bundesrepublik in eine Glaubwürdigkeits- und Autoritätskrise abgleiten, die die Herausbildung unterschiedlich extremistischer Haltungen zuließ.

$\mathrm{Zu}$ einem ersten Bruch in den Biographien von Thomas Weisbecker und Georg von Rauch kam es durch den, wenn auch nicht zeitgleich stattfindenden, jedoch konform begründeten Verweis von der Kieler Gelehrtenschule. Georg von Rauch besuchte ab 1958 die Kieler Gelehrtenschule und musste sie bereits im Jahr 1961 wegen disziplinarischer Schwierigkeiten wieder verlassen. Er machte daraufhin sein Abitur in Königsfeld im Schwarzwald. Danach kehrte er 1966 nach Kiel zurück und begann das Studium der Philosophie, Soziologie und Politologie an der CAU. Im Zuge seines Radikalisierungsprozesses, wechselte von Rauch im Herbst 1968 an die Freie Universität Berlin. Kurz darauf heiratete er die Künstlerin Ilse (Illo) Wittlich; ihre gemeinsame Tochter Yamin wurde am 22. März 1967 geboren. Im Sommer 1969 gab von Rauch sein Studium auf. Als Begründung schrieb er: „weil es mir unmöglich geworden ist, in

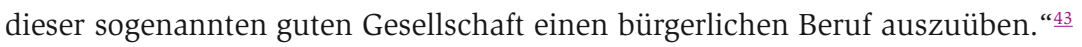

Thomas Weisbecker musste, nachdem es zu Konflikten zwischen ihm und einem Lehrer an der Kieler Gelehrtenschule gekommen war, diese 1967 verlassen. Er bestand im Juni 1968 sein Abitur in Karlsruhe und ging daraufhin für ein Studium der Soziologie und Theologie nach Frankfurt a. M. Weisbecker zog 1969 zu seinem älteren Bruder Lutz nach West-Berlin und wurde in diesem Umfeld bald darauf zu einem Aktivisten der linken Szene. Eine Freundschaft der beiden Professorensöhne entwickelte sich in Folge des Eintritts Weisbeckers in die von Georg von Rauch 1968 gegründete Wieland-Kommune und der sich daraus im Frühjahr 1969 formierenden ,Umherschweifenden Haschrebellen‘ $\underline{44}$ Mit einer Kerngruppe der Haschrebellen reisten Weisbecker und von Rauch im Juli 1969 in ein palästinensisches Ausbildungslager, um sich einer gründlichen politischen Schulung und einer soliden Ausbildung an der Waffe zu unterziehen. Als sie im Oktober des gleichen Jahres nach West-Berlin zurückkehrten, formierte sich die sogenannte „Palästina-Gruppe“ neu: Sie legte die „Hippie-Attitüden“ der Haschrebellen ab und gründete die ,Tupamaros West-Berlin“, die nun weit entschlossener den bewaffneten Kampf forderte. Innerhalb eines knappen Jahres verübten die West-Berliner Tupamaros mehr als 20 Brand- und Sprengstoffanschläge auf Banken und Kaufhäuser, Richter und Staatsanwälte. .5

Am 6. Februar 1970 wurde der Quick-Reporter Horst Rieck in seiner Berliner Wohnung von Mitgliedern der ,Tupamaros West-Berlin', unter ihnen auch Thomas Weisbecker und Georg von Rauch, überfallen und brutal zusammengeschlagen. Vorausgegangen war den Ereignissen ein Rieck zugeschriebener Artikel in der Illustrierten 


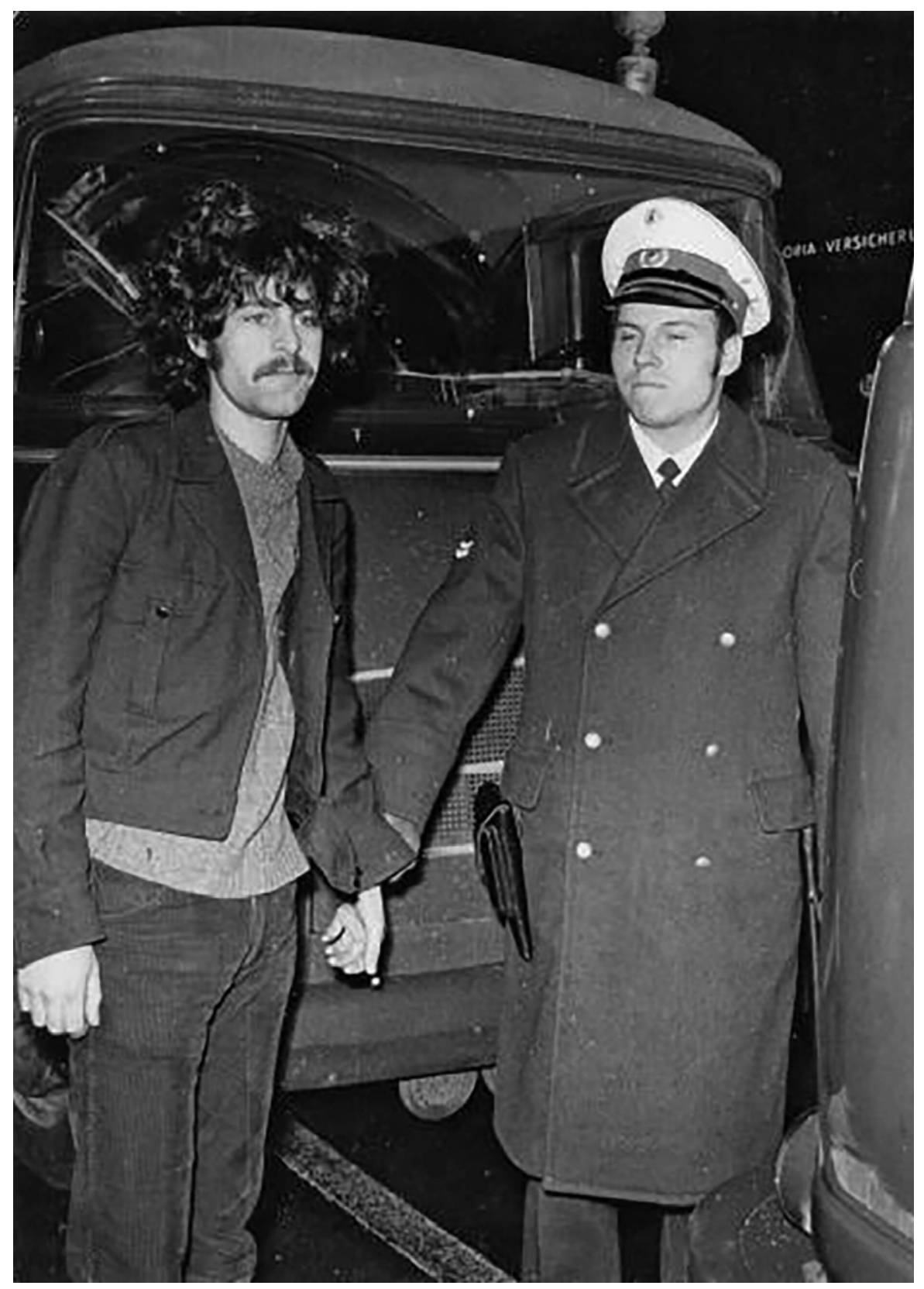

Abb. 7: Festnahme Thomas Weisbeckers.

(C) Tommy-Weisbecker-Haus, veröffentlicht unter der Lizenz CC-BY-NC 3.0 DE. 
Quick, von dem sich die linksradikale Szene verraten und verkauft fühlte. $\underline{46}$ Weisbecker und von Rauch wurden noch in der Wohnung Riecks von der Polizei verhaftet und am darauffolgenden Tag dem Haftrichter vorgeführt. Georg von Rauch kam direkt in die Untersuchungshaftanstalt nach Moabit, Thomas Weisbecker, zunächst wieder freigelassen, wurde am 20. Juli 1970 ebenfalls in Moabit inhaftiert. $\stackrel{47}{ }$ Beide mussten ein Jahr, zumeist unter verschärften Haftbedingen, auf ihre Hauptverhandlung warten. Nach Bekanntgabe der Haftverschonung Weisbeckers nutzten die beiden diese am 8. Juli 1971 für den so genannten „Verwechselungs-Go-Out“: Weisbecker, der von Rauch sehr ähnlich sah, verblieb an dessen Stelle im Gerichtssaal, während von Rauch den Saal verließ, als dies dem haftbefreiten Weisbecker vom Gericht erlaubt wurde. Am Tag darauf wurde Weisbecker zur Fahndung wegen Gefangenenbefreiung ausgeschrieben. Von Rauch lebte fortan in der Illegalität. ${ }^{48}$

Am 4. Dezember 1971, der Fahndungsdruck auf Georg von Rauch hatte sich durch zahlreiche weitere Aktionen stetig erhöht, starb dieser in einem Schusswechsel mit der Polizei in West-Berlin. Die genauen Umstände seines Todes sind bis heute ungeklärt. Die Eltern erfuhren vom Verlust ihres Sohnes aus der Tagesschau. ${ }^{49}$

Am 2. März 1972 wurde Thomas Weisbecker im Zuge einer Fahndungsaktion in Augsburg durch die Kugel eines Polizisten getötet. In diesem Fall fiel nur ein einziger Schuss, der einwandfrei der Waffe des Polizisten zugeordnet werden konnte. Auch bei Weisbecker erfuhren die Eltern vom Tod ihres Sohnes aus den Medien: Die Frankfurter Rundschau hatte sich bei Rotraud Weisbecker telefonisch gemeldet und um eine Stellungnahme zum Tod gebeten..$\frac{50}{}$ Später stellte sie Strafanzeige beim Landgericht Augsburg wegen vorsätzlicher Tötung. Die Ermittlungen der Staatsanwaltschaft wurden allerdings im August 1972 eingestellt.

\section{„Die traurige Weihnacht des Professors von Rauch“ Zu den überregionalen Reaktionen auf den Tod Thomas Weisbeckers und Georg von Rauchs}

In einem Artikel vom 22. Dezember 1971 mit gleichlautender Überschrift heißt es:

„Ihre Eltern machten Karriere als Professoren. Aber die Kinder dieser Eltern gehen den Weg der Gewalt. Sie töten! Sie sterben. So wie jetzt der Professorensohn Georg von Rauch in einem Gefecht mit der Polizei. [...] Und welches Elternpaar wird das nächste sein, das wie Professor von Rauch bei der Polizei anrufen wird: In welcher Leichenhalle liegt mein Sohn?“티 
Dieser reißerische Artikel steht stellvertretend für die nach dem Tod der beiden Professorensöhne einsetzende Flut an Mediendarstellungen, die mal mehr, mal weniger objektiv über die Geschehnisse berichteten. In einem Nachruf von Horst Bieber in Die Zeit vom 14. Januar 1972 schrieb dieser:

„Der Fall macht Schlagzeilen, [...]. Während Parteien und Publizisten darüber rechten, ob Polizeipräsident Hübner gehen muß oder ob Innensenator Neubauers Stuhl wackelt, ist das Opfer in Vergessenheit geraten. Kein Wunder, denn 24 Stunden nach dem physischen Tod war auch der Rufmord an Georg von Rauch perfekt. Gangster, Gewalttäter, Terrorist, Schießwütiger, Baader-Meinhof-Komplice, freigiebig verwendete Attribute in einer an Recht und Ordnung orientierten Presse, die dennoch geflissentlich den Grundsatz übersieht, daß ein Verdächtiger bis zum Urteil als unschuldig zu gelten hat.“드

Weiter heißt es, dass sich eine anonym gebliebene Kielerin an die Eltern von Rauchs gewandt hatte: „Wir danken unserem Herrgott, daß die Polizei ihren Sohn, diesen Mörder und Verbrecher, umgelegt hat; seine Komplicen kommen auch noch dran. “ $\underline{53}$

Als ein weiteres Beispiel für die starke Medienpräsenz veröffentlichte Der Spiegel im Januar 1972 unter dem Titel „Geschwärzte Hände“ Teile des Obduktionsberichts Georg von Rauchs und versuchte, zumindest den Tathergang und die Umstände des Todes sachlich zu erläutern. Der Vater des Ermordeten habe bereits Strafanzeige gegen „unbekannte Bedienstete des Landes Berlin gestellt. “ $\stackrel{54}{ }$ Eine Reaktion, die er selber in einem Brief an den Berliner FDP-Politiker Hermann Oxfort begründete: „[...] Es geht mir nicht um meinen toten Sohn allein, obwohl ich mich stets dagegen wenden werde, daß sein Bild völlig verzerrt wird. “ 55 Die Klage des Vaters wurde bereits im Mai 1972 abgewiesen.

Am 3. März 1972, einen Tag nach dem Tod Thomas Weisbeckers, wurde ein Sprengstoffanschlag auf das Landeskriminalamt in West-Berlin verübt, der als direkte Antwort auf dieses Ereignis, aber auch auf den Tod seiner Freunde Petra Schelm und Georg von Rauch, zu verstehen ist. Die ,Bewegung 2. Juni', die sich als Reaktion auf den Tod der beiden formiert hatte, verübte im Mai des Jahres einen weiteren Anschlag auf die Juristische Fakultät in West-Berlin, weil sie den Umgang mit dem Todesschützen Weisbeckers, der keine weiteren gerichtlichen Konsequenzen zu tragen hatte, nicht akzeptieren wollte. Sieben Tage später, am 12. Mai 1972, verübte das ,Kommando Thomas Weisbecker' der RAF einen Anschlag auf die Polizeidirektion in Augsburg. $\frac{56}{6}$ 


\section{„Lähmende Stummheit und Gleichgültigkeit“ Die Kieler Universität und ihr Umgang mit dem Tod der Professorensöhne}

Die 68er-Bewegung endete, so die aktuelle Forschungslage, mit dem Jahr 1970, dem Jahr, in dem sich der Bundesverband des Sozialistischen Deutschen Studentenbunds (SDS) in Frankfurt a.M. selbst auflöste und die große Anzahl an Massendemonstrationen langsam abnahm. $\frac{57}{}$ In Kiel, wie auch an anderen bundesdeutschen Hochschulstandorten, bildeten sich zahlreiche linksextreme K-Gruppen, Kommunisten wie die ,Rote Garde‘ oder ,Rote Zellen'. Sie forderten fortan, unter Rückgriff auf die Ressourcen der vorangegangenen Protestbewegungen, die Ordnung der Hochschule heraus. Damit waren die Konflikte und Spannungen innerhalb der Universität um studentische Mitbestimmungsrechte und strukturelle Reformen, wie sie bereits zu Beginn geschildert wurden, weiterhin tagesaktuell. So kam es an der Kieler Universität auch nachfolgend zu massiven Störungen und Auseinandersetzungen bei Senatssitzungen, bei der Eröffnung der Universitätstage, bei einzelnen Lehrveranstaltungen, was bis zu einem erneuten kompletten Zusammenbruch des Lehrbetriebs vom 26. bis 30. Juli 1972 führte. $\frac{58}{}$ Die Hochschulleitung bezog zu diesen Ereignissen in einer Pressemitteilung Stellung:

„Die organisierte Verhinderung dieser Lehrveranstaltungen reicht von der Störung durch Zwischenrufe bis zu massiven Maßnahmen, die studierwillige Studenten daran hindern, die Unterrichtsräume zu betreten und die Lehrveranstaltung $\mathrm{zu}$ besuchen. Hierdurch ist der Lehrbetrieb an der Universität zusammengebrochen. [...] Da die Universität keine rechtlichen und exekutiven Möglichkeiten besitzt, die Freiheit von Forschung und Lehre sicherzustellen, hat sie dem Kultusminister einen Bericht über Umfang und Art der Störungen

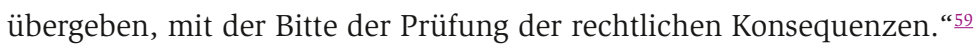

Die rechtliche Konsequenz lautete: „Anzeige wegen Hausfriedensbruch in mehreren Fällen“. 60 Als Reaktion belagerten etwa 50 Studierende den Weg zum Dienstzim-

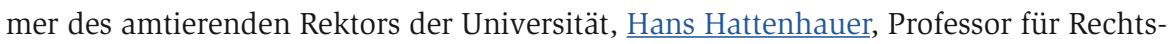
geschichte, Bürgerliches Recht und Handelsrecht, mit dem Ziel, dass dieser die Anzeigen gegen die studentischen Störer der Lehrveranstaltungen wieder zurücknehme. Während des Zwischenfalls kam es zu Tätlichkeiten zwischen den Studenten und dem Rektor, in deren Folge dieser zu Boden fiel. $\underline{\text {. }}$

Sicher sind auch die Auseinandersetzungen an der Kieler Universität im Kontext der bundesweiten Eskalation des linken Terrorismus der Jahre 1970 bis 1973 zu interpretieren. Der Tod der beiden Kieler Professorensöhne führte zu einer weiteren Eska- 
lation der Gewalt und schuf wiederum neue gewaltbereite Akteure. Diese Dynamisierungsschübe hatten sich seit der Erschießung Benno Ohnesorgs im Jahr 1967 bis hin zum Tod Thomas Weisbeckers und Georg von Rauchs 1971/72 immer wieder gezeigt und sich in ihrer Intensität gesteigert. Die Kieler Universität sah sich in diesen Krisenzeiten im Spannungsfeld zwischen den Reformen ihrer eigenen akademischen Strukturen, den darauf erfolgten Protesten der Studierenden und einer weiteren Radikalisierung des linken Terrorismus im Zuge der bundesweiten Entwicklungen. Alle drei Positionen schienen sich Anfang 1972 im direkten politischen, gesellschaftlichen und kulturellen Umfeld der Christiana Albertina zu fokussieren..$\underline{\text {. }}$

Ein Aktenvermerk der Universitätspressestelle vom 2. März 1972 gewährt einen Einblick in die Bemühungen des Rektorats, mittlerweile geleitet vom Professor für Pflanzenbau und Pflanzenzüchtung, Gerhard Geisler, die Ereignisse um den Tod von Georg von Rauch und Thomas Weisbecker weitestgehend aus dem regionalen Kontext zu isolieren. So lautete die offizielle Pressemitteilung:

„Veranlaßt durch die zahlreichen Anfragen beim Rektorat der ChristianAlbrechts-Universität Kiel teilt die UPS [Universitätspressestelle] mit, daß die bei den polizeilichen Festnahmeaktionen in Berlin und Augsburg tödlich verletzten Studenten Georg von Rauch und Thomas Weisbecker ebenso wie der in Hamburg festgenommene und durch Schüsse verletzte Manfred Grashof keine Studenten der Kieler Universität sind. Eine Beziehung zwischen den tragischen Ereignissen in Berlin und Augsburg sowie dem Schusswechsel in Hamburg und der Kieler Universität besteht nicht. Bei den Getöteten, denen Verbindungen zur Baader/Meinhof-Bande nachgesagt werden, handelt es sich um die Söhne der Kieler Professoren Dr. phil. Georg von Rauch und Dr. med. Ludwig Weisbecker [...]. Prof. Gerhard Geisler bittet in seiner Eigenschaft als Rektor der Kieler Universität, das tragische Schicksal der betroffenen Eltern zu respektieren und bei der Befriedung des spontanen Informationsbedürfnisses die Grenzen angemessener Zurückhaltung zu beachten.“䇇

Signifikant an der Pressemitteilung der Universität ist zunächst die Wortwahl in Bezug auf die Benennung des ,Baader/Meinhof-Komplexes' als ,Baader/Meinhof-Bande' oder ,Baader/Meinhof-Gruppe'. Die Hochschulleitung hatte sich in ihrer öffentlichen Bekanntgabe zum Tod der Professorensöhne für den Ausdruck Bande entschieden und sich damit eindeutig positioniert: Sie sah in dem ,Baader/Meinhof-Komplex' eine kriminelle Vereinigung, in deren direktes Umfeld sie die beiden Professorensöhne begrifflich stellte. Auffallend ist weiterhin, dass der Satz „Eine Beziehung zwischen den tragischen Ereignissen [...] und der Kieler Universität besteht nicht“ offenkundig 
versucht, alle möglichen Verbindungen des bundesweiten linken Terrors mit einer regionalen Identifikation zu unterbinden. Erst im unteren Absatz wird eine Beziehung zur Kieler Hochschule durch die zu den Kieler Professoren bestehenden familiären Verbindungen eingeräumt: „Bei den Getöteten [...] handelt es sich um die Söhne der Kieler Professoren. “ Im darauffolgenden Absatz allerdings folgt von Seiten des Rektorats wiederum eine Unterbindung der Kommunikation mit den Medien unter dem Vorwand, es gelte „[...] bei der Befriedung des spontanen Informationsbedürfnisses die Grenzen angemessener Zurückhaltung zu beachten“. Die Hochschulleitung hatte sich mit ihrer Pressemitteilung dazu entschlossen, die Rolle der CAU in Bezug auf die Konflikte um die Personen Thomas Weisbeckers und Georg von Rauchs in der Öffentlichkeit nicht näher zu verorten. Mit der Aussage, dass keiner der Getöteten - aktuell - Student der Kieler Universität sei, hatte sie sich wahrheitsgemäß geäußert. Dass Georg von Rauch allerdings dort erst knapp zwei Jahre zuvor sein Studium absolviert hatte, wurde in keinem weiteren Zusammenhang erwähnt.

In einem weiteren Aktenvermerk vom 3. März 1972 unterbindet die Pressestelle anfangs die Weitergabe von Informationen zur Person Ludwig Weisbeckers. Wörtlich heißt es: „Auf Anruf von Frau Hagelein vom Hamburger Abendblatt, die Auskünfte über Herrn Prof. Weisbecker haben wollte, wurde zunächst die Aussage verweigert.“ Erst nach der Erstellung eines festen Antwortenkatalogs zu wenigen bestimmten Daten wie den Jahren der Promotion und Habilitation wurden Fragen beantwortet. Auskünfte über „den weiteren beruflichen Werdegang vor der Zeit als Medizinischer Direktor in Karlsruhe, Umfang und Art der wissenschaftlichen Veröffentlichungen und zum beruflichen Werdegang, eventuell Studium, der Kinder“, wurden hingegen verweigert. $\stackrel{64}{ }$ Scheinbar verfolgte die Universität, so lässt der Aktenvermerk vermuten, eine Strategie der Informationsverweigerung statt einer -offenlegung. Sie bemühte sich, die Verbindungen zu der bundesweiten Eskalation des linken Terrors sowie zu den Geschehnissen um Thomas Weisbecker und Georg von Rauch herunterzuspielen, und wo sie offenkundig wurden, möglichst zu isolieren. Sicher muss in die Beurteilung dieser Haltung auch das potentielle Verantwortungsbewusstsein der Universitätsleitung gegenüber den Professoren von Rauch und Weisbecker und ihren Funktionen innerhalb des Lehrkörpers berücksichtigt werden. In Bezug auf die Öffentlichkeit bleibt aber das Bild, dass sich die Kieler Universität als Landesinstitution durch Pressestelle und Rektorat für einen Weg der Verdunklung entschieden hatte.

In der Personalakte zu Professor von Rauch findet sich ein Schreiben des damaligen Rektors der CAU, Professor Gerhard Geisler, das sein Bestreben bestätigt, dem Tod Georg von Rauchs jun. keinen tiefgreifenden Stellenwert zu geben: 
„Zu dem tragischen Verlust, der Sie betroffen hat, darf ich namens der Universität und auch persönlich Ihnen und Ihrer Gattin tiefempfundenes Beileid übermitteln. Es ist sicher eine Versuchung, der die Jugend unterliegt, sich in Gegensatz zur Gesellschaft zu stellen und diesen Gegensatz mit letzter persönlicher Konsequenz zu vertreten..$\underline{65}$

Mit der Formulierung „Versuchung der Jugend“, die der Rektor nicht nur persönlich, sondern auch im Namen der Institution Universität wählt, wird der Tod des Sohnes zu einer Art Bagatelle transformiert. Die Umstände werden heruntergespielt, um ihnen jede Form weiterreichender Bedeutung zu entziehen.

In einer Beiakte des Kultusministeriums bat Professor von Rauch um eine Beihilfe zur Bestattung seines Sohnes. Diese wurde abgelehnt. Zur Erklärung hieß es: „Da die Zahlung des Kindergeldzuschlages für Ihren Sohn Georg bereits mit Ablauf des Monats Mai 1970 eingestellt wurde, [...] ist es leider nicht möglich, Ihnen aus Anlaß des Sterbefalls Ihres Sohnes eine Beihilfe zu bewilligen. “흐 Die Entscheidung der Universität ist aus rein objektiver und rechtlicher Perspektive sicher einwandfrei, aus einer subjektiven und zwischenmenschlichen Sicht jedoch hätte die Universität eine Ausnahmeregelung in Betracht ziehen können.

Am 10. Dezember 1971 wurde Georg von Rauch jun. auf dem Parkfriedhof Eichhof in Kiel beerdigt, die Grabrede hielt der Flensburger Theologe und Pädagoge Theodor Christiansen. ${ }^{67}$ In der Rede versuchte der Pastor, der die Familie von Rauch nicht kannte, das Schicksal eines jungen Mannes darzustellen, den „subjektiv empfundenes und objektiv erlebtes Unrecht zum Protest gegen die Gesellschaft getrieben hatte, der vielleicht nicht immer das Richtige getan, aber das Rechte gewollt habe!?“. Zudem bekannte er: „Ich kann mich mit Georg von Rauch und seinen Freunden nicht identifizieren [...], aber ich solidarisiere mich mit ihnen. “토 Ausführlich ging Christiansen auf die Haftbedingen in Moabit ein und sah in den verschärften Haftbedingen Parallelen zu den Konzentrationslagern in den Diktaturen Mittel- und Südamerikas.

Aufgrund dieser Grabrede konnte der Pastor, der seine Tätigkeit als Religionslehrer am Flensburger Goethe-Gymnasium für ein Studium der Geschichte in Kiel unterbrochen hatte, vorerst nicht wieder, wie beantragt, in den Schuldienst zurückkehren. Am 21. Juni 1972 schrieb der Präsident des Landesschulamtes, Heinrich Laufer, an das Landeskirchenamt: „Leider kann ich Ihrer Bitte, Herrn Pastor Christiansen nach Ablauf seiner Beurlaubung am 31. Juli 1972 im Schuldienst des Landes Schleswig-Holstein weiter zu beschäftigen, nicht entsprechen.“으 Der Vorwurf lautete, Christiansen habe den kriminellen Charakter der ,Baader-Meinhof-Bande’ - so die Wortwahl - bestritten, ihr „verbrecherisches Handeln verharmlost und in einem gewissen Umfang zu rechtfertigen versucht“. ${ }^{70}$ Erst nach Verhandlungen des Schleswiger Bischofs Alfred 
Petersen mit den Verantwortlichen der Landesregierung konnte Pastor Christiansen im Oktober 1972 seinen Schuldienst wieder aufnehmen..$\underline{1}$

Auch wenn die Reaktionen auf die Grabrede und ihre Konsequenzen in keinem direkten Zusammenhang zur Universität stehen, sondern ausschließlich die Entscheidungen des Landes Schleswig-Holstein widerspiegeln, so zeichnen sie doch ein authentisches Bild der damaligen Stimmungslage. Die Geschehnisse offenbaren ebenfalls, mit welcher Intensität die Landesregierung bemüht war, öffentlich geäußerte Solidarisierungs- und Sympathiebekundungen schnellstmöglich zu unterbinden und - falls geschehen - ihnen Konsequenzen folgen zu lassen.

Unmittelbar als Reaktion auf den Tod Thomas Weisbeckers hatten am 13. März 1972 die Studentenpastoren Dieter Andresen und Manfred Kamper sowie der Theologe und Universitätsprediger Professor Joachim Scharfenberg ein Flugblatt der Universitätskirche unterzeichnet, dass sie in einer Auflage von 300 Exemplaren als Beitrag zur Diskussion innerhalb der Hochschule verteilten. Der Inhalt setzte sich mit der gesellschaftlichen Situation auseinander, in der einerseits der Protest einiger Gruppen zur Gewaltanwendung eskalierte und sich andererseits der „Gegendruck bis zur aggressiven Hysterie“ steigerte. So heißt es:

„Georg von Rauch und Thomas Weisbecker sind erschossen worden. [...] Das Vorgehen der Staatsgewalt findet die Zustimmung von über 90 Prozent der Bevölkerung. Man erwartet die Lösung des Problems von der physischen Vernichtung der anarchistischen Gruppen. Man ist erleichtert, wenn es wieder einen von ihnen erwischt hat. [...] Der Mechanismus allgemeiner Verblendung funktioniert auch bei uns. Er besteht darin, daß eine kranke Gesellschaft sich Gruppen schafft, auf die sie ihre ungelösten Konflikte überträgt, um sie leichter bekämpfen zu können, in der trügerischen Hoffnung, diese dadurch loszuwerden.“

Am Ende folgt der Aufruf: „Lassen Sie sich nicht anstecken von einer aggressiven Hysterie, die die Freiheit in Gefahr bringt! Widerstehen Sie dem Sog der Resignation, die zur achselzuckenden Gewöhnung an solche Ereignisse führt! “프

Der Text traf weit über die Grenzen der Kieler Universität auf ein breites Echo und erzeugte neben zahlreichen Äußerungen der Zustimmung - Dieter Andresen wies in einer zweiten Stellungnahme zum Flugblatt auf einen ausdrücklichen Dank der Eltern Georg von Rauchs hin - auch Kritik. $\frac{73}{}$ Viele kirchennahe Zeitungen druckten das Flugblatt, andere distanzierten sich von seinem Inhalt: „Es ist gut so, daß dieses Papier nicht in die breite öffentliche Diskussion gelangte, denn es enthält Formulierungen und Gedankengänge, die teils unrichtig, teils emotional überhöht sind [...].“푸 
Die wohl schärfste Kritik am Inhalt des Flugblattes äußerte der damalige Ministerpräsident Gerhard Stoltenberg, der seit 1971 die Regierungsverantwortung in Schleswig-Holstein trug, in einem Brief vom 27. März 1972 an die Kieler Kirchenleitung. „In einer völligen Verdrehung von Ursachen und Wirkung“, so warnte Stoltenberg, „offenbaren die Sätze einen Geist der Feindseligkeit gegenüber der großen Mehrzahl unserer Mitbürger und unser freiheitlich-demokratischen Staatsordnung, der den Schlußapell nach Verständigung und Diskussion zwischen politischen Gegnern völlig überlagert. [...] Es ist deshalb unannehmbar, wenn jetzt Gewalttäter als Opfer einer angeblich ,kranken Gesellschaft' freigesprochen werden und stattdessen der Staat und die Mehrheit seiner Bürger verantwortlich gemacht werden sollen. “포

In seiner erläuternden Stellungnahme „Zum Flugblatt der Kieler Universitätskirche“ nahm Dieter Andresen Bezug auf die Kritik des Ministerpräsidenten: „Wir können nur noch einmal auf die Intention hinweisen: sie ist zuerst seelsorgerlich. Angesichts der lähmenden Stummheit und Gleichgültigkeit gegenüber dem Tod der beiden Kieler Studenten [anders als in der Pressemitteilung des Rektorats wurden die Getöteten von den Autoren des Flugblatts als Kieler Studenten bezeichnet] waren wir der Meinung, hier nicht einfach schweigen zu dürfen." $\underline{\text { 6 }}$ Neben dem Kommentar zum Tod der Professorensöhne, einer möglichen Erklärung und dem Versuch eines angemessenen Umgangs damit, offenbaren die Autoren des Flugblatts damit noch eine weitere Absichtsebene: Es sollte die „lähmende Stummheit und Gleichgültigkeit“ aufbrechen, die sich scheinbar in der Bevölkerung Kiels ausgebreitet hatte. Das Flugblatt illustriert demnach, dass die von der Universität gewollte und praktizierte Isolierung der Ereignisse aus dem übergeordneten Kontext ihre Wirkung nicht verfehlt hatte. Die Angehörigen der Hochschule und mit ihnen die gesamte Kieler Öffentlichkeit nahmen scheinbar keinen Anteil am Schicksal der beiden Professorensöhne, weil durch eine mangelnde Informationspolitik und einer maßgeblichen Unterdrückung von Identifikationsmöglichkeiten des Rektorats die Geschehnisse auf gesellschaftlicher, kultureller wie emotionaler Ebene entkoppelt wurden. Wer Stellung bezog, sah sich, wie Pastor Christiansen, die Initiatoren des Flugblattes oder der studentische Protestbewegung, mit dem Rechts- und Gewaltmonopol des Staates / des Landes konfrontiert.

Die „lähmende Stummheit und Gleichgültigkeit“ gegenüber dem gewaltsamen Tod der Professorensöhne sollte sich noch lange hinziehen. Auch noch Jahrzehnte danach - Prof. Rauch starb 1991 - vermied es die Universität peinlichst genau, mögliche Verbindungen zwischen dem bundesdeutschen Terrorismus mit den regionalen Entwicklungen zuzulassen. So finden sich keine Hinweise im Nachruf auf Professor von Rauch in der Universitätszeitschrift Christiana Albertina, die über das persönliche Schicksal der Familie und die polarisierende Geschichte Aufschluss geben könnten. $\cdot \underline{77}$ Auch im Fall Weisbecker gibt es in den Nachrufen keine Anhaltspunkte zum tragi- 
schen Verlust seines Sohnes oder zu den näheren Umständen der Ereignisse: Die Kieler Universität dankt ihm in ihrer Todesanzeige vom 14. Juni 1979 in den Kieler Nachrichten einzig und allein dafür, dass er als Rektor „,in bewegter, schwerer Zeit für die Christiana Albertina Verantwortung trug“.르 Der Nachruf in der Universitätszeitschrift würdigte daneben noch seine vielfältige Schaffenskraft und sein Wirken, sparte aber ebenfalls jegliche persönliche Bezugnahme zu den Ereignissen der Jahre 1970 bis 1972 aus. $\underline{79}$

\section{Schlussbetrachtung}

Der vorliegende Beitrag zum Umgang mit akademischem Traditionsbewusstsein und politischer Radikalisierung an der CAU trägt zu einem vertieften Verständnis und einem differenzierten Blick auf die Krisenzeit zwischen 1969 und 1972 bei, indem die Geschehnisse aus zwei unterschiedlichen Perspektiven beleuchtet wurden: So konnten die studentischen Protestbewegungen, die sich aus dem Kontext der universitären Strukturreformen - wie dem Immatrikulationsverfahren und den neuen Hochschulgesetzen - entwickelt hatten, auf einer regionalen Ebene verortet werden. Die bis dato traditionsbewusste Kieler Alma Mater befand sich durch die Pläne einer neuen Hochschul- und Studienreform im Prozess der Neuordnung. Ihre akademische Selbstbestimmung wurde vom Kultusministerium durch die Einführung einer neuen Rechtsordnung und von der Studierendenschaft, die ein erhöhtes Maß an Mitbestimmungsrechten forderte, zunehmend eingeschränkt. Wie stark sich die Konflikte und Auseinandersetzungen zwischen der studentischen Protestbewegung und der Hochschulleitung der CAU ausgebreitet und verdichtet hatten, konnte am Beispiel des Rektoratsjahrs von Ludwig Weisbecker 1969/70 gezeigt werden. Dieser regionale Fokus hilft dabei, den Umgang der Universitätsleitung mit dem bundesdeutschen Terror, hier personifiziert durch die Professorensöhne Thomas Weisbecker und Georg von Rauch, besser zu verorten.

Die zweite Perspektive beleuchtete die politische Radikalisierung und mit ihr den linken Terrorismus auf einer bundesweiten Ebene, hier konkretisiert anhand der Biographien von Thomas Weisbecker und Georg von Rauch. Die beiden Kieler Professorensöhne, die bereits vor ihrem gewaltsamen Tod zu den Stars der linksradikalen Szene in West-Berlin gehörten, wurden nach ihrem Tod in ganz Deutschland zu bewunderten Ikonen des bewaffneten Kampfes. Dadurch, dass sie ihre ideologischen Vorstellungen und Ansprüche mit dem Leben bezahlten, kam es zu weiteren Dynamisierungsschüben innerhalb der radikalen Linken und damit zu einer fortlaufenden Eskalation von Gewalt. 


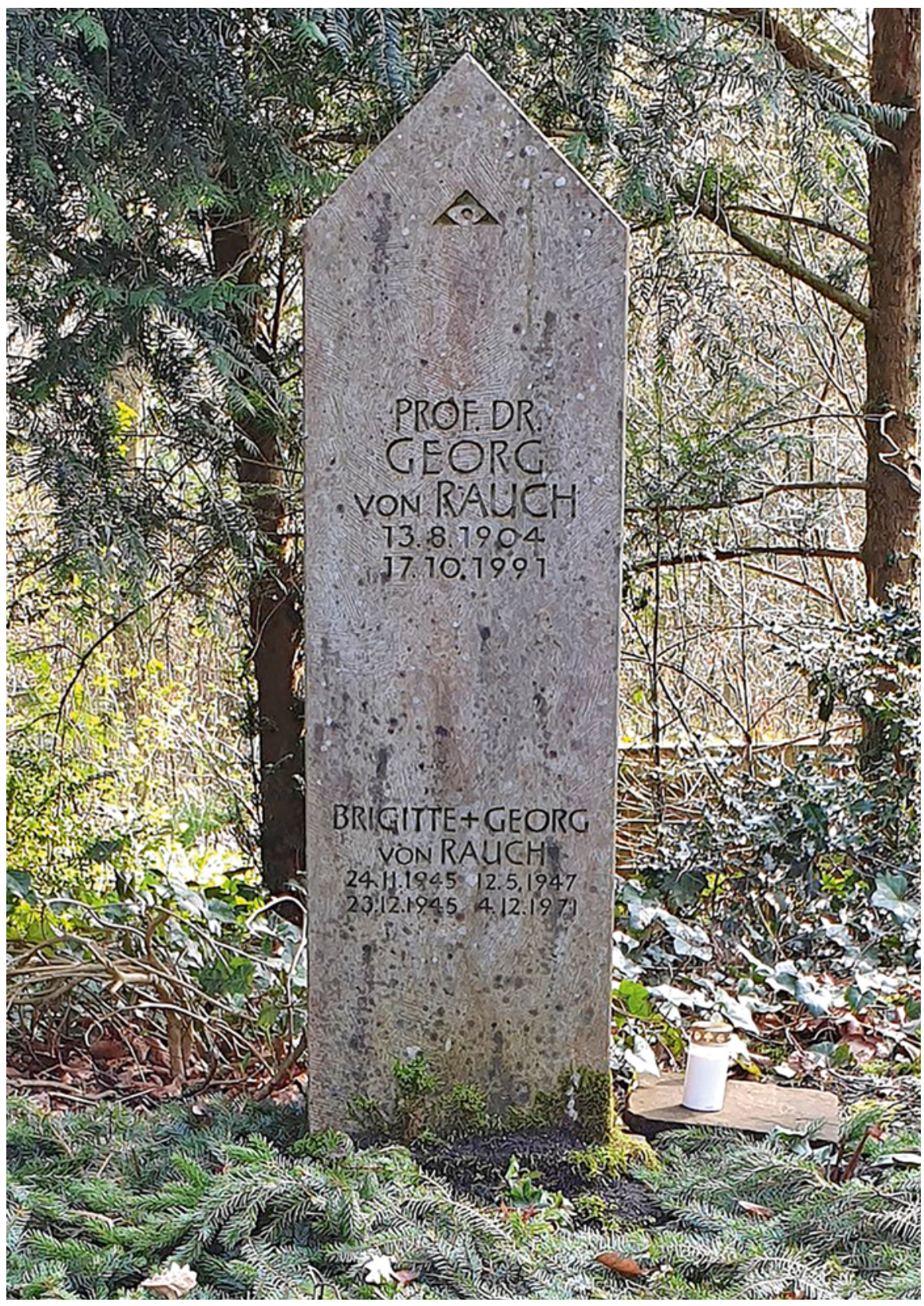

Abb. 8: Grabstein von Prof. Dr. Georg von Rauch auf dem Eichhof Friedhof in Kiel.

(C) 2019 Mwehle, veröffentlicht unter der Lizenz CC-BY-SA 4.0, Quelle: https://de.wikipedia.org. 
Besonders wichtig erscheint es bei der erfolgten Betrachtungsweise darauf hinzuweisen, dass sich die beiden beschriebenen Blinkwinkel nicht separat betrachten lassen, weil sie sich gegenseitig bedingen und ergänzen. So lassen sich regionale Radikalisierungsprozesse sicher nicht von den bundesweiten Entwicklungen des linken Terrorismus abgrenzen und umgekehrt. Als Schnittpunkte der regionalen und überregionalen Entwicklungen der linken Radikalisierung lassen sich beispielsweise die Auflehnung gegen die Elterngeneration, die politischen Verhältnisse, die unzureichende Aufklärung der nationalsozialistischen Vergangenheit und allgemein die Ablehnung von Kapitalismus, Faschismus und Imperialismus konstatieren. Für die hier vorgestellten Kieler Ereignisse der Jahre 1969 bis 1972 und dem sich daraus entwickelnden Spannungsfeld lässt sich festhalten, dass sich das Konfliktpotential sowohl aus kulturell-ideologischen Aspekten als auch aus den im universitären Kontext erfahrbaren institutionellen Problemen zusammensetzte.

Die ausgewählten Quellen zur Kieler Universitätsgeschichte konnten anschließend verdeutlichen, wie sich die Hochschulleitung im Zuge einer direkten Konfrontation zu dem bundesweiten linken Terrorismus positionierte. Mit dem Tod der beiden Kieler Professorensöhne und der daraus resultierenden weiteren Eskalation der Gewalt trat der linke Terrorismus in das direkte Umfeld der CAU. Um einer erneuten Zuspitzung der ohnehin bereits konflikt- und spannungsreichen Verhältnisse an der Universität entgegenzuwirken, entschloss sich die Hochschulleitung aus strategischen Gründen gegen Transparenz und Kontextualisierung der gewaltsamen Todesfälle der beiden ehemaligen Studenten sowie zur bewussten Isolierung der tragischen Ereignisse. Vielleicht fürchtete die Universität durch eine offene Kommunikation in weitere Konflikte und Auseinandersetzungen mit den Studierenden, den Sympathisanten innerhalb der Bevölkerung und den Kritikern in den eigenen Reihen zu geraten. Die Christiana Albertina befand sich zu diesem Zeitpunkt, wie bereits erwähnt, in einem Prozess der Neuordnung weg von der Abgeschlossenheit einer internen Ordinarienhierarchie hin zu mehr Öffentlichkeit und Mitbestimmungsrechten der Studentenschaft. Durch die Planung und Umsetzung der neuen Hochschulgesetze sollte auch das Kultusministerium stärker in die Autonomie der Hochschule eingreifen.

Somit ließe sich die Haltung des Rektorats gegenüber dem Tod der beiden Professorensöhne aus den zu diesem Zeitpunkt noch bestehenden tradierten akademischen Normen und Werten erklären, die die ideologischen Vorstellungen und Ansprüche der linken Gruppierungen partout ablehnten. Oder sie erklärt sich aus der Tatsache heraus, dass die Hochschulleitung neben ihrem aktuellen Transformationsprozess keine weiteren Auseinandersetzungen und Konflikte aufkommen lassen wollte und somit versuchte, sich gegen die bundesweiten Entwicklungen des Linksradikalismus abzuschirmen. Damit agierte sie, teils bewusst teils unbewusst, ganz im Sinne der damali- 
gen schleswig-holsteinischen Landesregierung, die ebenfalls auf eine strikte Unterbindung jeglicher Solidarisierungsversuche - wie an den Reaktionen gegen den Pastor Christiansen oder das Flugblatt der Kieler Universitätskirche gesehen - mit den linken Gruppierungen setzte.

\section{Dr. Swantje Piotrowski ist Wissenschaftliche Mitarbeiterin für die Digital Humanities am Historischen Seminar der CAU.}

Kontakt: s.piotrowski@email.uni-kiel.de

\section{Anmerkungen}

Der Beitrag ist unter der Creative-Commons-Lizenz Namensnennung 4.0 international veröffentlicht. Den Vertragstext finden Sie unter: https://creativecommons.org/licenses/by/4.0/deed.de. Bitte beachten Sie, dass einzelne, entsprechend gekennzeichnete Teile des Werks von der genannten Lizenz ausgenommen sein bzw. anderen urheberrechtlichen Bedingungen unterliegen können.

1 Ludwig Weisbecker, Rektoratsbericht für das akademische Jahr 1969/70, in: Christiana Albertina 9 (1970), S. 5-11, hier: S. 5.

$\underline{2}$ Zur Schreibweise des Namens Weisbecker, der häufig in der Literatur und in den Quellen mit "ss" geschrieben wird, äußert sich Ludwig Weisbecker in einem Schreiben an das Kultusministerium folgendermaßen: „Natürlich haben Sie recht, der Geburtsname wird leider mit einem "s" geschrieben, obwohl das Unsinn ist, denn der Name Weißbecker ist ein Zunftname, also der eines Bäckers, der Weißbrot backt. Wenn man inn mit einem "s" schreibt, dann würde es bedeuten, der weise, der kluge Bäcker, was man von mir nicht mit der an Sicherheit grenzender Wahrscheinlichkeit behaupten kann." Demnach schrieb Weisbecker seinen Namen stets mit "SS", laut Geburtsurkunde wurde dieser, wie in den folgenden Ausführungen geschehen, allerdings nur mit "s" geschrieben. Vgl. Schreiben Ludwig Weisbecker an das Kuratorium der CAU, 28.12.1967, Landesarchiv Schleswig-Holstein (LASH), Abt. 811, Nr. 12178

3 Weisbecker, Rektoratsbericht (wie Anm. 1), S. 11.

$\underline{4}$ Vgl. ebd.

$\underline{5}$ Zur Geschichte des linksradikalen Terrorismus und Topologie des RAF-Terrorismus siehe immer noch das zweibändige Standardwerk von Wolfgang Kraushaar (Hg. ), Die RAF und der linke Terrorismus, 2. Bde., Hamburg 2006.

$\underline{6}$ Für weiterführende Informationen zur 68er-Bewegung an der Kieler Universität siehe Rainer S. Elkar, Studieren in Kiel. Eine historisch-politische Zeitreise von den Anfängen bis zur Gegenwart (Sonderveröffentlichungen der Gesellschaft für Kieler Stadtgeschichte/77), Husum 2015.

7 Vgl. dazu Stefan Bichow, Die Universität in den 1960er Jahren. Ordnungen einer akademischen Institution in der Krise (Kieler Werkstücke, Reihe H/3), Frankfurt a. M. 2013. 
$\underline{8}$ Vgl. Christian-Albrechts-Universität zu Kiel, Mitteilungen des Rektors, Kiel 1970.

$\underline{9}$ Vgl. Verein für Politik, Geschichte und Dokumentation Zeitzeichen e.V. (Hg.), Die APO in Kiel. Die antiautoritäre Revolte 1967 bis 1972 (Arbeitshefte aus dem Kieler Archiv für neue soziale Bewegungen/1), Kiel 1992, S. 66.

10 Vgl. Schreiben des Dekans der Philosophischen Fakultät an den Kultusminister des Landes Schleswig-Holsteins mit Betreff „Dienstaufsichtsbeschwerde”, 20.11.1969, LASH, Abt. 811, Nr. 12437. Aus einem Aktenvermerk geht hervor, dass am 6. Januar 1970, nach einem Gesprächstermin zwischen dem Dekan, Prof. Ott und Ludwig Weisbecker, die Dienstaufsichtsbeschwerde zurückgenommen wurde. Wörtlich heißt es zur Begründung „[...], daß der reibungslose Ablauf des Vorlesungsbetriebes im Interesse der Universität wie auch der Gesellschaft liege." Vgl. ebd.

11 Vgl. Schreiben des Kultusministers des Landes Schleswig-Holsteins zur Anerkennung des Unfalls vom 25.11.1969 als Dienstunfall vom 4. April 1970, LASH, Abt. 811, Nr. 12178.

12 Vgl. H.A., Sorgen um Kiels Rektor. Unter die Gürtellinie, in: Die Zeit (5.12.1969), abrufbar unter: https://www.zeit.de/1969/49/unter-die-quertellinie (09.02.2020,17:26 Uhr).

13 Weisbecker, Rektoratsbericht (wie Anm. 1), S. 11.

14 Ebd.

$\underline{15}$ Gerhard Knuth und Urs Stahl, Autorität ist Persönlichkeit plus Leistung, in: Kieler Nachrichten (22.3.1969), S. 12.

16 Weisbecker, Rektoratsbericht (wie Anm. 1), S. 6

17 Die Ordinarienuniversität wird im vorliegenden Zusammenhang, nach Kraushaar, als eine überkommen geltende hierarische Ordnung der Universität definiert. Vgl. Wolfgang Kraushaar, „Unter den Talaren ...". Ein Gespräch mit Wolfgang Kraushaar über den Antiakademismus der Studentenbewegung, in: Mittelweg 36. Zeitschrift des Hamburger Instituts für Sozialforschung 26 (2017) 4/5, S. 168-189, hier S. 168.

18 Vgl. dazu Bichow, Die Universität Kiel (wie Anm. 7), S. 158 f. Die Studierenden kritisierten die Ordinarienuniversität in ihrer Struktur und forderten entsprechend dem Humboldt'schen Bildungsideal nicht nur die Einheit von Forschung und Lehre, sondern auch die Einheit von Lehrenden und Lernenden. Vgl. Kraushaar, „Unter den Talaren ..." (wie Anm. 17), S. 172. Zum generellen Wandel des Hochschulbetriebs von einer reinen Lehranstalt zu einer Forschungseinrichtung vgl. Swantje Piotrowski, Sozialgeschichte Kieler Professorenschaft 1665-1815. Gelehrtenbiographien im Spannungsfeld zwischen wissenschaftlicher Qualifikation und sozialen Verflechtungen (Kieler Schriften zur Regionalgeschichte/2), Kiel/Hamburg 2018.

19 Vgl. fachlicher Lebenslauf von Ludwig Weisbecker, LASH, Abt. 811, Nr. 12437.

20 Vgl. Arbeitszeugnis der Chemischen Werke Albert, 11.5.1950, ebd.

$\underline{21}$ Schreiben des Landesamtes für Wiedergutmachung Freiburg, 24.6.1955, LASH, Abt. 811, Nr. 12178.

22 Vgl. persönlicher Lebenslauf Ludwig Weisbeckers, LASH, Abt. 811, Nr. 12437.

23 Karin König, Zwei Ikonen des bewaffneten Kampfes. Leben und Tod Georg von Rauchs und Thomas Weisbeckers, in: Die RAF und der linke Terrorismus/1, hg. von Wolfgang Kraushaar, Hamburg 2006, S. 430-472, hier: S. 434

24 Vgl. persönlicher Lebenslauf Ludwig Weisbeckers, LASH, Abt. 811, Nr. 12178.

$\underline{25}$ Im Haushalt der Familie Weisbecker wohnte neben den zwei Söhnen und einer Tochter noch ein im Jahr 1969 aufgenommenes Pflegekind, vgl. Personalblatt von Ludwig Weisbecker, ebd.

$\underline{26}$ Vgl. Berufungsverhandlungen Ludwig Weisbeckers, LASH, Abt. 811, Nr. 12437.

$\underline{27}$ Schreiben Ludwig Weisbeckers an den Kultusminister des Landes Schleswig-Holstein, 19. 2.1979, LASH, Abt. 811, Nr. 12178.

28 Vgl. König, Zwei Ikonen (wie Anm. 23), S. 439.

$\underline{29}$ Ludwig Weisbecker überlebte nicht nur die KZ-Gefangenschaft, sondern engagierte sich im Lager als Arzt mit tiefer humanistischer Prägung. In einem Schreiben des „Headquarter Buchenwald Concentration Camp, Office of the Medical Director" vom 25. Mai 1945 wurde Ludwig Weisbecker "a high sense of medical ethics" bescheinigt. Vgl. LASH, Abt. 811, Nr. 12178.

30 Rotraut Weisbecker, Unveröffentlichtes Manuskript, Stadtarchiv Kiel, S. 52, zitiert nach: König, Zwei Ikonen (wie Anm. 23) , S. 438

31 Vgl. ebd, S. 439

32 Diese und alle folgenden Informationen zur Person Georg von Rauchs wurden seiner Personalakte im LASH, Abt. 47, Nr. 6930 entnommen

33 Die Abteilung Fremde Heere war eine im Mai 1917 begründete Dienststelle zur Bewertung der Feindlage des deutschen Heeres, die im Ersten Weltkrieg im Großen Generalstab des deutschen Heeres eingerichtet wurde. Vgl. hierzu grundlegend Magnus Pahl, Fremde Heere Ost. Hitlers militärische Feindaufklärung. Berlin 2012.

34 Michael Gerleff, Georg von Rauch. 1904-1991. Würdigung und Auswahlbibliographie, in: Berichte und Forschungen. Jahrbuch des Bundesministeriums für ostdeutsche Kultur und Geschichte/1, München 1993, S. 123-145, hier: S. 132.

35 Vgl. König, Zwei lkonen (wie Anm. 23), S. 440.

$\underline{36}$ Noch heute bestehen die beiden Jugendzentren, das Georg-von-Rauch-Haus, vgl. http://www.rauchhaus1971. de/ und das Thomas-Weisbecker-Haus, vgl. https://www.tommyhaus.org/ (beide 10.02.2020, 14:00 Uhr).

37 Vgl. Michael Sontheimer und Peter Wensierski (Hg.), Berlin. Stadt der Revolte, Berlin 2018, hier S. 85-91.

$\underline{38}$ Zur Debatte um eine wissenschaftlich gesicherte Terrorismusdefinition vgl. Wolfgang Kraushaar, Zur Topologie des RAFTerrorismus, in: Die RAF und der linke Terrorismus/1 (wie Anm. 23), S. 13-64, hier: S. 29-31.

39 Der linke Terrorismus und sein Netzwerk ist jedoch ein vielschichtiges und höchst dynamisches Phänomen, das hier angesichts gebotener Kürze nicht Gegenstand der Untersuchung ist, sodass die zahlreichen Strukturen und Formierungen der unterschiedlichen terroristischen Gruppen an dieser Stelle nicht näher erläutert werden können. Sie müssen teilweise als Wissensbasis vorausgesetzt werden, teilweise stehen sie auch nicht in einem direkten Zusammenhang zu den zu Beginn aufgeworfenen Fragen. Ebenfalls können die näheren Umstände und die einzelnen Entwicklungen, die zu Beginn der Radikalisierung von Thomas Weisbecker und Georg von Rauch geführt haben, im vorliegenden Kontext nicht näher analysiert werden. Dies alles sind Fragen, die bereits in anderen Aufsätzen und Publikationen aufgegriffen und ausreichend thematisiert wurden. Zu den Gründen des Radikalisierungsprozesses von Weisbecker und von Rauch formuliert König in ihrem Beitrag "Zwei Ikonen" verschiedene Theorien, die sie in ihren Ausführungen auf eine Reihe persönlicher Interviews, unter anderem mit der Schwester Thomas Weisbeckers, Verena Weisbecker, stützt. 
40 Kraushaar, Zur Topologie (wie Anm. 38), S. 24 f.

41 Theodor Christiansen, Ansprache am Sarge von Rauchs am 10.12.1971 in Kiel, in: Junge Kirche. Eine Zeitschrift europäischer Christen 33 (1972), H. 3, S. 155-160, hier S. 156.

42 Ludwig Weisbecker war Mitglied der New York Academy of Science, Inhaber der Juan-Finlay-Medaille für große Verdienste auf dem Gebiet der Hämatologie sowie der Silbernen Jubiläums-Plakette der Universität Freiburg und Mitglied mehrerer inund ausländischer Fachgesellschaften. 1965 ernannte ihn die Van-Swieten-Gesellschaft zum Korrespondierenden Mitglied. Darüber hinaus gehörte er dem Kultursenat der Stadt Kiel jahrelang an. Vgl. LASH, Personaldeckblatt, Abt. 811, Nr. 12178.

43 Horst Bieber, Unser Leben zählt nichts, in: Die Zeit (14.1.1972), abrufbar unter: https://www.zeit.de/1972/02/unser-lebenzaehlt-nichts/komplettansicht (09.02.2020,17:26 Uhr).

44 Vgl. Sontheimer/Wensierski (wie Anm. 37), S. 85-91.

$\underline{45}$ Vgl. Wolfgang Kraushaar, Die Tupamaros West-Berlin, in: Die RAF und der linke Terrorismus/1, hg. von dems., Hamburg 2006, S. 448-453.

46 Vgl. König, Zwei Ikonen (wie Anm. 23), S. 448-453.

47 Ludwig Weisbecker benachrichtigt das Innenministerium über die Verhaftung seines Sohnes „wegen politischer Vergehen” und vermutet weiter eine Verurteilung mit Freiheitsstrafe. Vgl. Schreiben Ludwig Weisbeckers an das Innenministerium des Landes Schleswig-Holsteins, 11.12.1970, LASH, Abt. 811, Nr. 12178.

48 Vgl. Heinrich Böll, Die Erschießung des Georg von Rauch. Dokumente anläßlich des Prozesses gegen Klaus Wagenbach, Berlin 1976

49 Vgl. König, Zwei lkonen (wie Anm. 23), S. 462.

$\underline{50}$ Vgl. ebd, S. 465

$\underline{51}$ Die traurige Weihnacht des Professors von Rauch: Ein Sohn erschossen. Ein Sohn inhaftiert, in: praline aktuell Nr. 52 (22.12.1971)

$\underline{52}$ Vgl. Bieber, Unser Leben zählt nichts (wie Anm. 43).

$\underline{53}$ Vgl. ebd.

$\underline{54}$ Geschwärzte Hände, in: Der Spiegel (10.1.1972),abrufbar unter: https://www.spiegel.de/spiegel/print/d-43019380.html (09.02.2020,17:26 Uhr).

$\underline{55}$ Brief des Vaters Georg von Rauch an den FDP-Politiker Hermann Oxfort, in: Extradienst 6 (1972), Nr. 4, S. $6 f$.

$\underline{56}$ Vgl. Tobias Wunschik, Die Bewegung 2. Juni, in: Die RAF und der linke Terrorismus/1, (wie Anm. 23), S. 531-561.

$\underline{57}$ Vgl. dazu Tilman Fichter und Siegward Lönnendonker (Hg.), Kleine Geschichte des SDS: Der Sozialistische Deutsche Studentenbund von Helmut Schmidt bis Rudi Dutschke, Bonn 2008.

$\underline{58}$ Vgl. Elkar, Studieren in Kiel (wie Anm. 6), S. 143-162.

$\underline{59}$ Pressemitteilung der Universität, 27. 6.1972, LASH, Pressemitteilungen 1964-1973 Abt. Acc82/07, U2 Reg. 48 F5 1.

60 Ebd.

$\underline{61}$ Vgl. Pressemitteilung der Universität, 27.11.1972, ebd.

62 Noch im Jahr 1973 kritisierte der Kulturpolitische Beirat des SPD-Landesverbandes Schleswig-Holstein in einem offenen Brief zur Lage der Universität das Verhalten des Rektorats gegenüber den anhaltenden studentischen Protesten scharf. Der Beirat klagte an, "dass das Rektorat für ein so aktuelles Thema keine Form gewählt hat, in der die Pluralität der hochschulpolitischen Meinungen Ausdruck gefunden hätte“. Weiter heißt es: „Hier wäre während der Universitätstage [zuvor war es während der Eröffnungsveranstaltung der Universitätstage 1973 zu Störaktionen von ca. 50 Studierenden gekommen] zu diskutieren gewesen, ob nicht das Verhalten der Landesregierung selbst jene Entwicklungen provoziert, zu deren Bekämpfung angeblich das Gesetz erforderlich ist." Vgl. Offener Brief des SPD-Landesverbandes an das Rektorat der CAU, 29. 01.1973, LASH, Abt. Acc82/07, U2 Reg. 48F5 1.

63 Aktenvermerk, 2.3.1972, ebd.

64 Aktenvermerk, 3.3.1972, ebd. und LASH, Abt. 811, Nr. 12178.

$\underline{65}$ Schreiben des Rektors an Georg von Rauch, 10. Dezember 1972, LASH, Abt. 47, Nr. 6930.

66 Ebd.

$\underline{67}$ In einer im Jahr 2018 erschienenen Reihe des Schleswig-Holsteinischen Zeitungsverlags (SHZ) mit dem Titel "Die Revolte und der Norden" heißt es in einem der Artikel, dass beide Professoren-Söhne in Kiel begraben wurden. Vgl. Frank Jung, Flugblatt-Affäre um Terroristen-Tod, in: shz (7.5.2018).

68 Christiansen, Ansprache am Sarge (wie Anm. 41), S. 158.

69 Horst Bieber, Grabrede für Georg von Rauch. Anstößige Predigt - Ist Pastor Christiansens Laufbahn gefährdet?, in: Die Zeit (13.10.1972), abrufbar unter: https://www.zeit.de/1972/41/anstoessige-predigt/komplettansicht (09.02.2020, 17:26 Uhr).

$\underline{70}$ Art. ,Pastor Christiansen soll wieder unterrichten', in: Hamburger Abendblatt (11.10.1972).

$\underline{71}$ Vgl. König, Zwei lkonen (wie Anm. 23), S. 462.

72 Dieter Andresen ( $H g$.) , Kirche am Montag. Kieler Beispiele öffentlicher Kommunikation (Konkretionen - Beiträge zur Lehre von der handelnden Kirche/18), Hamburg 1973, S. 111-124, hier: S. 111.

$\underline{73}$ Vgl. Ders., Zum Flugblatt der Kieler Universitätskirche, in: Ebd., S. 122 f.

$\underline{74}$ Ders., Nordelbischer Konvent, Kirchliche Mitarbeiter Nr. 5, 1972, in: Ebd., S. 120.

$\underline{75}$ Ders., Brief des Ministerpräsidenten Gerhard Stoltenberg an Bischof Dr. Hübner, 27.3.1972, in: Ebd., S. 115 f.

$\underline{76}$ Andresen, Zum Flugblatt (wie Anm. 73), S. 124.

$\underline{77}$ Vgl. Peter Nitsche, Nachruf auf Georg von Rauch, in: Christiana Albertina 34 (1992), S. 294-295.

$\underline{78}$ Todesanzeige Ludwig Weisbeckers, in: Kieler Nachrichten (15.6.1979).

$\underline{79}$ Vgl. Jörg Dieter Herrlinger, Nachruf auf Ludwig Weisbecker, in: Christiana Albertina 11 (1979), S. 197-199. 